\title{
Expression of autophagic and ubiquitin- proteasome proteins in the peripheral nervous system after nerve injury
}

\author{
Sithiporn Agthong*, Marisa Rodtayoy, Atitaya Roumwong
}

\begin{abstract}
Background: Autophagy and ubiquitin-proteasome (UPS) are two main degradation systems for intracellular proteins. They are essential for homeostasis of neurons during normal and pathological conditions, but their changes after nerve injury remain unclear.

Objective: To examine the protein expression of autophagy and UPS in the dorsal root ganglia (DRG), including intact and injured sciatic nerves after crush injury in rats.

Methods: Left sciatic nerve crush was done in all Wistar rats and the specimens were removed at 1, 3, 7, and 14 days after injury. Expression of the autophagic (Beclin-1 and p62) and UPS proteins [muscle ring finger-1 (MuRF1) and ubiquitinated proteins] was measured using Western blot analysis.

Results: Expression of p62 was significantly increased in the injured versus intact sciatic nerves on day 1 and day 7 $(P<0.05$ and $P<0.01$, respectively). There was a trend toward higher expression of Beclin- 1 on the crushed nerve. In the DRG, expression of p62 and Beclin-1 was not significantly different between the two sides. Expression of MuRF1 and ubiquitinated proteins was not significantly different between the left and right DRG. The low quantity of MuRF1 and high variations in the ubiquitinated protein levels in the nerve prevented further analysis.

Conclusions: These results indicated the induction of autophagy with accumulation of autophagosomes in the nerve, but not DRG, after nerve injury. Future studies on the effects of the autophagic changes and the precise activity of UPS in nerve trauma are crucial.
\end{abstract}

Keywords: autophagy; nerve injury; proteasome; ubiquitin

Autophagy and ubiquitin-proteasome (UPS) are two major systems that are responsible for intracellular protein degradation. Macroautophagy, the most studied autophagy pathway, degrades large proteins in the membrane and organelles, whereas the UPS degrades small short-lived proteins. Autophagy (referred to macroautophagy here) involves the formation of autophagosome surrounding the protein target and fusion with lysosome for final degradation [1]. Several components are involved in the autophagosome formation, particularly autophagy-related gene (ATG) proteins, Beclin-1 and p62 have been widely used as markers of autophagic activity. In the UPS, target proteins are conjugated with ubiquitin

*Correspondence to: Sithiporn Agthong, Department of Anatomy, Faculty of Medicine, Chulalongkorn University, Bangkok 10330, Thailand, e-mail: Sithiporn.A@chula.ac.th

Department of Anatomy, Faculty of Medicine, Chulalongkorn University, Bangkok 10330, Thailand

¿ Open Access. ๑ 2019 Agthong et al., published by Sciendo. (c) BY-NC-ND This work is licensed under the Creative Commons Attribution NonCommercial-NoDerivatives 4.0 License. 
using ubiquitin ligases to become ubiquitinated proteins which are then transferred to and degraded by $26 \mathrm{~S}$ proteasome [2]. Ubiquitin ligases, for example Atrogin-1, muscle ring finger-1 (MuRF1) including levels of ubiquitinated proteins are used to indicate the UPS activity.

These degradation systems are particularly essential for homeostasis of neurons because abnormal proteins tend to accumulate throughout the lifespan of neurons. Evidence indicates that autophagy $[3,4]$ and UPS $[5,6]$ are impaired and linked to accumulated protein aggregates in neurodegenerative diseases such as Alzheimer's disease and Parkinson's disease. Nevertheless, existing data regarding their changes in nervous tissue injury are incomplete. Increased activity of autophagy was observed in brain and spinal cord injuries [7-10]. Activated autophagy was also shown after cranial and spinal nerve injuries [11-13]. However, the changes in the dorsal root ganglia (DRG) and nerve segments adjacent to the injury site have not been studied. As for the UPS, data are controversial. Ubiquitin mRNA, but not protein, was increased after motor axon injury [14], whereas higher level of ubiquitinated proteins was shown by the study [15]. Studies of gene expression reported both increased [16] and decreased [17] expression of UPS-related genes after spinal nerve injury.

Concerning the information above, the spatial and temporal changes of enhanced autophagic activity after nerve injury have not been fully elucidated. Moreover, the UPS activity after nerve injury remains inconclusive. Therefore, this study aimed to clarify these issues by studying the expression of protein markers of the autophagy and UPS in the DRG including the nerve proximal and distal to the injury site at various time points after sciatic nerve injury.

\section{Materials and methods}

\section{Animal surgery}

Left sciatic nerve crush under inhalation anesthesia using isoflurane was done on 28 male Wistar rats weighing 200-250 g from the National Laboratory Animal Center, Mahidol University. The sciatic nerve was exposed at the mid-thigh level and crushed using a fine arterial clamp for $30 \mathrm{~s}$, according to the previous study [18]. Epineurial suture with Ethilon 8/0 was done to mark the crush site. The skin wound was then sutured with Ethilon 4/0 and the rats were allowed to recover.

During the experiment, the rats were housed on a $12 \mathrm{~h}$ lightdark cycle with access to food and water ad libitum. The room temperature was maintained at $25 \pm 2{ }^{\circ} \mathrm{C}$. The experiment was approved by the Institutional Animal Care and Use Committee, Faculty of Medicine, Chulalongkorn University (Certificate of
Approval No. 24/2558) and carried out in accordance with The Animals for Scientific Purposes Act 2015, Thailand.

\section{Tissue collection}

At $1,3,7$, and 14 days after the crush surgery, seven rats were randomly sacrificed by overdose of isoflurane and subsequent cardiac puncture. This number of rats per time point was based on our pilot study. After sacrifice, bilateral 4th-6th lumbar DRG, proximal and distal stumps (indicated by the epineurial suture) of sciatic nerve were removed immediately and snapfrozen on dry ice. These specimens were then kept at $-70^{\circ} \mathrm{C}$ until the Western blot analysis was processed.

\section{Western blot analysis}

Sciatic nerves and pooled L4-L6 DRG were homogenized and prepared as described in detail in the previous study [18]. The concentration of protein was determined using the Bramhall assay [19]. Sodium dodecyl sulfate polyacrylamide gel electrophoresis (SDS-PAGE) was performed on 25 and $30 \mu$ g protein for the DRG and sciatic nerve, respectively. The protein was then transferred to nitrocellulose membrane using a semidry electroblotter. The membranes were blocked in casein solution and incubated in the primary antibodies to autophagic proteins: p62 and Beclin-1 (1:500, Pierce) and UPS proteins: MuRF1 (1:1,000, Pierce) and ubiquitinated proteins (1:1,000, Millipore) for $24 \mathrm{~h}$ at $4^{\circ} \mathrm{C}$. The membranes were then incubated with the biotinylated secondary antibody (Vector) for $30 \mathrm{~min}$ followed by avidin and biotinylated horseradish peroxidase (HRP) (Vectastain ABC reagent, Vector) for $30 \mathrm{~min}$. Then, diaminobenzidine (DAB) (DAB substrate kit for peroxidase, Vector) was applied on the membranes until dark-brown color developed. The specific bands were scanned, and densities were analyzed using Image-Pro Plus software. Average value of each protein for each nerve segment/DRG was calculated from all seven rats. The amount of protein loading was examined by probing the blots with antibody to beta-actin (1:1,000, Cell Signaling).

\section{Statistical analysis}

One-way analysis of variance (ANOVA) with Tukey's Honestly significant difference as a post hoc comparison was employed to compare means of different time-points after crush injury, whereas Student's t-test was used for a comparison between two sides at the same time point. These tests were done using SPSS for Windows version 23. Statistically significant differences were considered when $P<0.05$. 


\section{Results}

\section{Expression of autophagic proteins}

At 1, 3, and 7 days after crush, expression of p62 was increased in both proximal and distal stumps of left (injured) compared with right (intact) sciatic nerves with significant

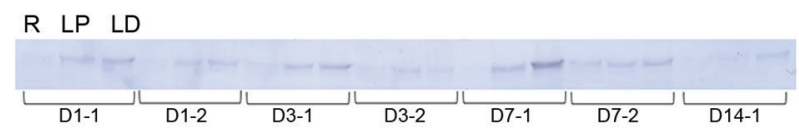

R LP LD
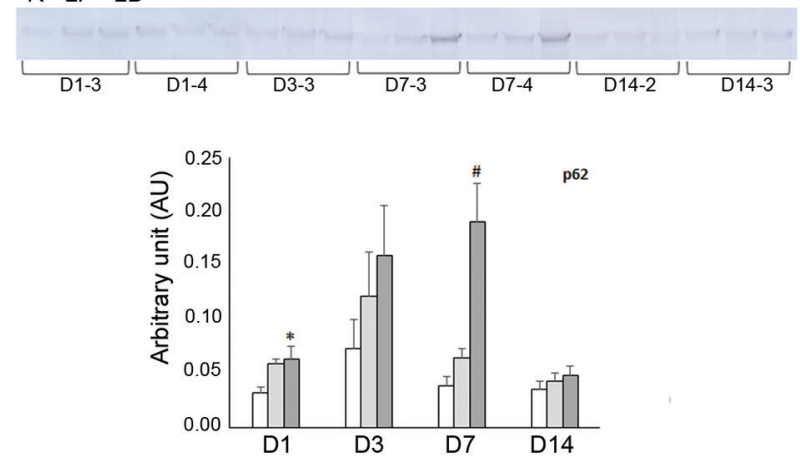

Figure 1. p62 protein in the right (R) (white bar), left proximal (LP) (light gray bar), and left distal (LD) (dark gray bar) sciatic nerves of rats sacrificed at day 1 (D1), day 3 (D3), day 7 (D7), and day 14 (D14) after nerve crush ( $n=7$ for each time point). Representative immunoblots are shown above. The bar chart demonstrates the average protein level at each time point. Error bars represent SEM. ${ }^{*} P<0.05$ vs. $R,{ }^{\#} P<0.01$ vs. $R$.

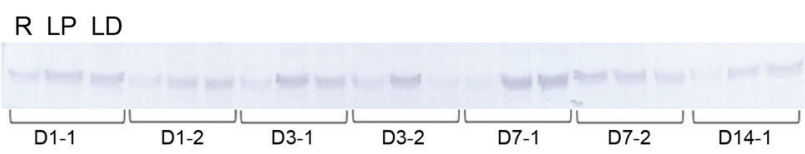

R LP LD
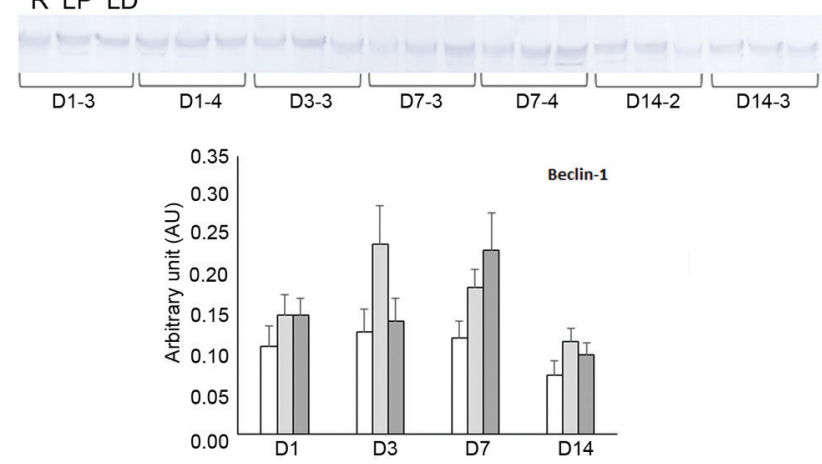

Figure 2. Beclin-1 protein in the right (R) (white bar), left proximal (LP) (light gray bar), and left distal (LD) (dark gray bar) sciatic nerves of rats sacrificed at day 1 (D1), day 3 (D3), day 7 (D7), and day 14 (D14) after nerve crush ( $\mathrm{n}=7$ for each time point). Representative immunoblots are shown above. The bar chart demonstrates the average protein level at each time point. Error bars represent SEM. differences between the left distal versus right on day 1 and day 7 ( $P<0.05$ and $P<0.01$, respectively; Figure 1). Furthermore, there was a trend toward higher expression of Beclin-1 on the left versus right sciatic nerves, especially on day 3 and day 7 although no statistical significance was observed (Figure 2). In the DRG, p62, and Beclin-1, expression was not significantly different between the left and the right DRG at any time point (Figures 3 and $\mathbf{4}$ ).

\section{Expression of ubiquitin-proteasome proteins}

MuRF1 expression was not significantly different between the left and right DRG at any time point (Figure 5). Due to the high variable expression of ubiquitinated proteins in the DRG of rats sacrificed at the same time point, left/right ratio in each rat was calculated and used for comparisons. The ratio was close to 1 at all time points, indicating minimal difference between sides (Figure 6). At all time points, the differences were not statistically significant although a trend toward higher value on day 3 was noted. In the sciatic nerve, expression of MuRF1 was barely detectable. In addition, many inconsistent bands of ubiquitinated proteins were observed among rats even from the same time points. Therefore, quantitative data of MuRF1 and ubiquitinated proteins were not obtained.

Expression of beta-actin was not significantly different between samples indicating the equal loading of total protein (Figure 7).

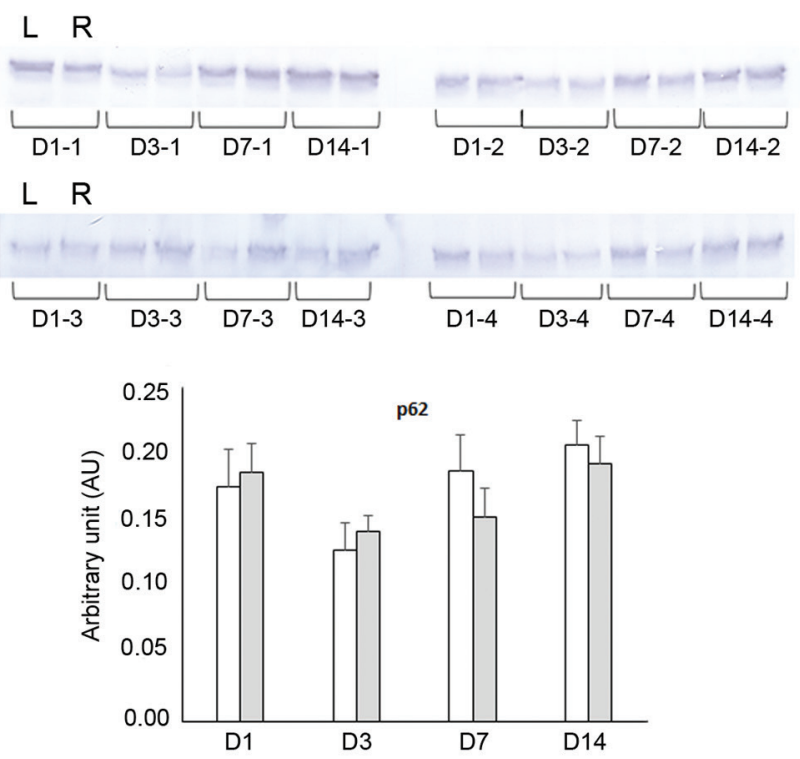

Figure 3. p62 protein in the right (white bars) and left (light-gray bars) L4-6 dorsal root ganglia of rats sacrificed at day 1 (D1), day 3 (D3), day 7 (D7), and day 14 (D14) after nerve crush ( $n=7$ for each time point). Representative immunoblots are shown above. The bar chart demonstrates the average protein level at each time point. Error bars represent SEM. 
L R

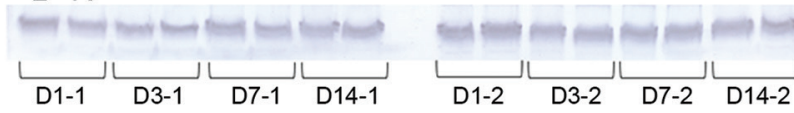

L R
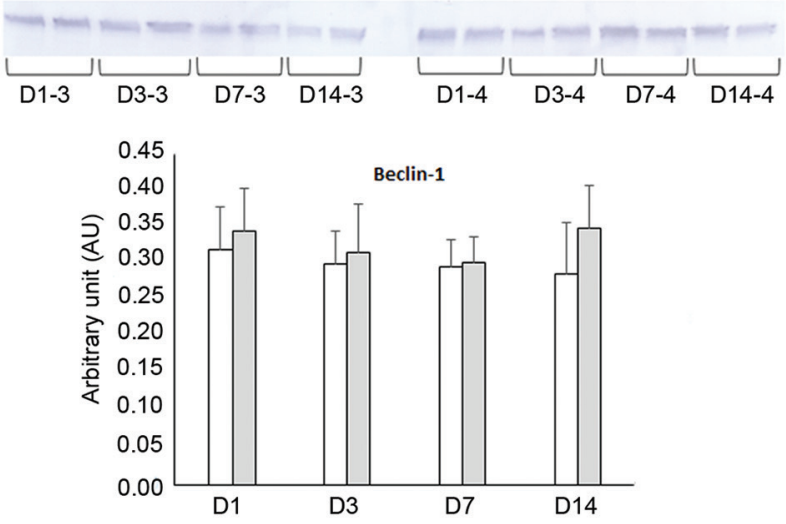

Figure 4. Beclin-1 protein in the right ( $R$, white bars) and left (L, gray bars) L4-6 dorsal root ganglia of rats sacrificed at day 1 (D1), day 3 (D3), day 7 (D7), and day 14 (D14) after nerve crush ( $n=7$ for each time point). Representative immunoblots are shown above. The bar chart demonstrates the average protein level at each time point. Error bars represent SEM.

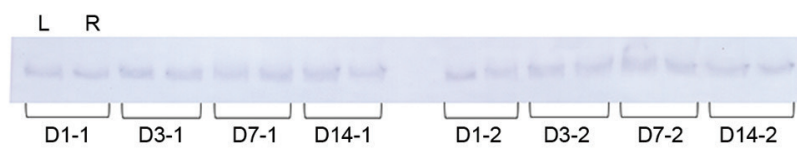

L R

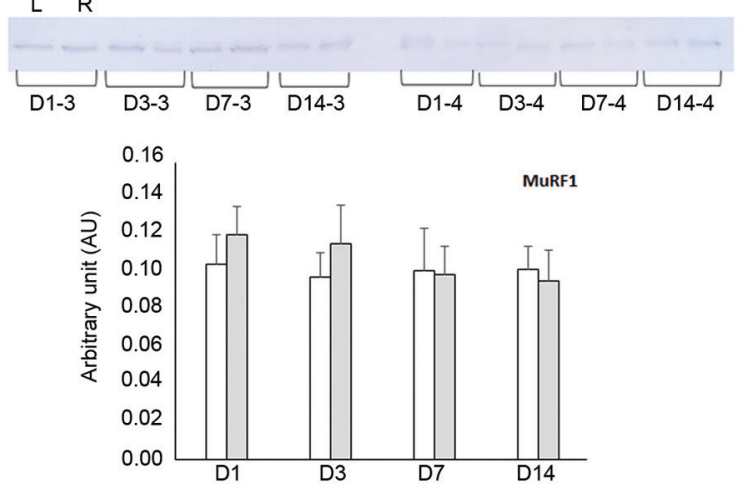

Figure 5. Muscle ring finger-1 protein in the right ( $R$, white bars) and left (L, left bars) L4-6 dorsal root ganglia of rats sacrificed at day 1 (D1), day 3 (D3), day 7 (D7), and day 14 (D14) after nerve crush ( $n=7$ for each time point). Representative immunoblots are shown above. The bar chart demonstrates the average protein level at each time-point. Error bars represent SEM.

\section{Discussion}

Our results showed that the expression of p62 was significantly upregulated in the injured sciatic nerve both proximal and distal to the crush site. This change was started at day 1 and disappeared at day 14. Despite without statistical
L R

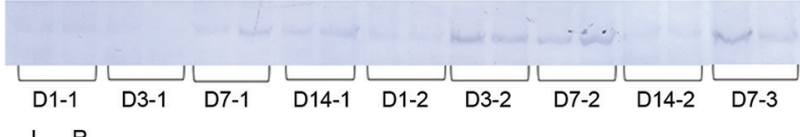

L R
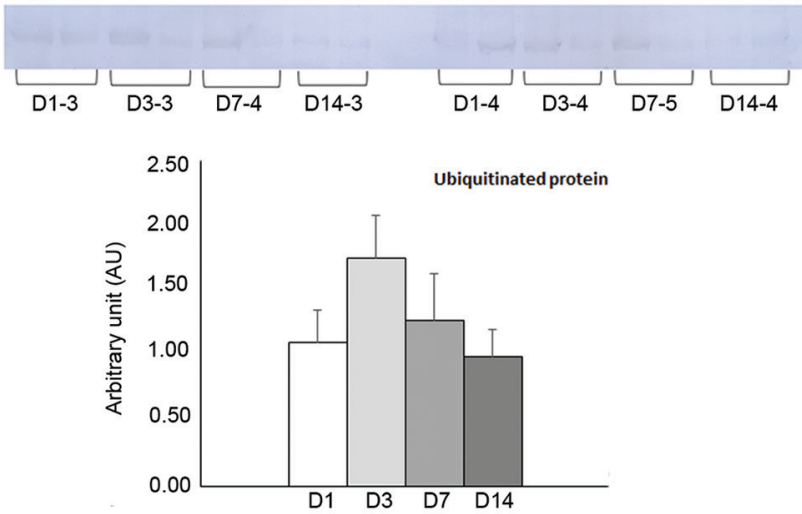

Figure 6. Ubiquitinated protein in the right $(\mathrm{R})$ and left $(\mathrm{L}) \mathrm{L} 4-6$ dorsal root ganglia of rats sacrificed at day 1 (D1), day 3 (D3), day 7 (D7), and day 14 (D14) after nerve crush ( $n=7$ for each time point). Representative immunoblots are shown above. The bar chart demonstrates the left/ right ratio at each time point. Error bars represent SEM.

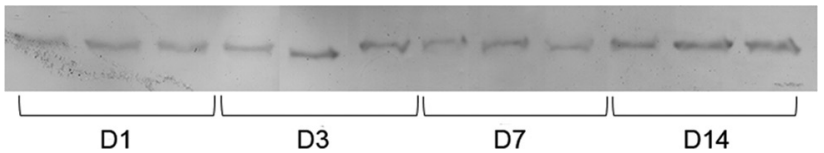

Figure 7. Representative immunoblot of dorsal root ganglia from rats sacrificed at day 1 (D1), day 3 (D3), day 7 (D7), and day 14 (D14) after nerve crush, probed for beta-actin.

significance, the expression of Beclin-1 tended to increase at the similar time points as seen in the p62. Increased $\mathrm{p} 62$ likely indicates accumulation of autophagosomes as its level decreases when proteins within autophagosomes are degraded. In contrast, minor increase in Beclin-1 might suggest slight activation of autophagosome formation. The similar changes in the expression of Beclin-1 and p62 were reported by Berliocchi et al. [20]. They found that spinal nerve ligation caused accumulation of autophagosomes. Another study also showed the enhanced autophagy and increased number of autophagosomes in the injured nerve 1 week following crush [13]. The other studies reported similar findings of activated autophagy after cranial or spinal nerve injuries [11, 12, 21]. According to the results of our present and previous studies, it is likely that nerve injury not only activates autophagosome formation in some degree but also causes accumulation of autophagosomes. Because we did not observe any alteration in the $\mathrm{p} 62$ or Beclin- 1 expression in the DRG throughout the study interval, these changes might occur locally in the injured nerve. This view was consistent 
with the results of the previous study showing that the autophagosomes are generated mainly in the axons [22]. In addition, Jang et al. [23] also demonstrated that autophagy was induced in Schwann cells after nerve injury.

In contrast to the autophagy, we did not find robust evidence of altered activity of the UPS after nerve injury. Savedia and Kiernan [14] reported the increased expression of ubiquitin mRNA, but not protein in motor neurons within 4 weeks after axotomy. However, another study found the increased levels of ubiquitination on day 1 after nerve injury [15]. Microarray studies also yielded conflicting results. $\mathrm{Hu}$ et al. [16] observed the elevated expression of UPS-related genes in motor neurons after nerve injury. However, another study showed reduced expression of the UPS genes in the similar model [17]. The reason of inconsistent findings among studies remains unclear but might be due to different techniques in measuring the UPS activity.

The mechanisms underlying enhanced autophagy and autophagosome accumulation following nerve trauma remain unclarified. It is conceivable that axonal degeneration after injury likely causes massive protein degradation, which requires higher activities of autophagy and UPS. Nevertheless, as discussed above, the UPS activity after nerve trauma is still controversial. Therefore, it seems that the autophagy is so far the main degradation system activated after nerve injury. The activated autophagy may be beneficial but it also occurs with autophagosome accumulation. In normal conditions, the autophagosomes generated in the distal axon are retrogradely transported to the proximal axon where final protein degradation occurs [22]. Induction of autophagy along with autophagosome accumulation was observed in neurodegenerative diseases [22]. The accumulation was likely due to the impaired axonal transport. After nerve injury, the axons are damaged and the axonal transport is thus blocked. This may explain our present and the previous studies' findings of autophagosome accumulation in the axons after nerve trauma. Consistent with this view, we found markedly elevated expression of p62 in the nerve distal to the crush site compared with those in the proximal and uninjured nerves, especially on day 7 . The autophagosomes generated by induced autophagy accumulate in the distal axons since the retrograde transport is blocked at the injury site.

The role of activated autophagy and autophagosome accumulation after nerve trauma is also unclear. Jang et al. [23] demonstrated that Schwann cells employed the autophagy to clear myelin debris. Regarding axons, evidence from a few studies suggests the beneficial roles. Rodriguez-Muela and Boya [11] found that optic nerve transection induced autophagy and apoptosis of retinal ganglion cells. When further stimulating the autophagy with rapamycin, the number of apoptotic cells significantly decreased. On the contrary, when the autophagy was inhibited by deletion of ATG4 gene, the apoptosis increased. These data indicate the beneficial effect of enhanced autophagy after optic nerve injury. Another study also reported the favorable effect of autophagy after sciatic nerve injury [13]. The autophagy was activated after injury and after treatment with rapamycin, motor recovery and morphology of nerve regeneration were improved compared with the control group. Inhibition of autophagy with 3-methyladenine resulted in worst outcomes. However, the effects of accumulated autophagosomes in the distal nerve have not been investigated. At least, our study demonstrated that this accumulation was temporary as the expression of p62 decreased to the level of intact nerve on day 14. In addition, as autophagy is also induced in Schwann cells for myelin clearance, reduction in p62 expression might reflect the complete clearance process. Further studies are required to confirm this hypothesis.

Taken together, our study showed that p62 expression was significantly elevated in the injured sciatic nerve within 7 days after crush, indicating the increased number of autophagosomes. The expression of Beclin-1 was slightly higher in the injured nerve at the similar time points, suggesting modest activation of autophagy. These changes were not seen in the DRG. In contrast, the UPS activity determined by the expression of MuRF1 and ubiquitinated proteins was not changed after nerve injury. These data confirmed the findings of the previous reports and added the temporal and spatial information of the autophagic changes after nerve trauma. Gaining more knowledge of the precise roles of autophagy and UPS in peripheral nerve injury will help to design specific treatments for this condition.

Author contributions. SA contributed substantially to the conception and design of this study. SA, MR, and AR contributed substantially to the acquisition of data. SA and MR analyzed and interpreted the data. SA drafted the manuscript. MR and AR contributed substantially to its critical revision. All the authors approved the final version submitted for publication and take responsibility for the statements made in the published article.

Acknowledgments. This work was supported by Rachadaphiseksomphot Fund 2016 (RA59/024) from the Faculty of Medicine, Chulalongkorn University.

Conflict of interest statement. The authors have completed and submitted the International Committee of Medical Journal Editors Uniform Disclosure Form for Potential Conflicts of Interest. None of the authors disclose any conflict of interest. 


\section{References}

[1] Mizushima N, Komatsu M. Autophagy: renovation of cells and tissues. Cell. 2011; 147:728-41.

[2] Tai HC, Schuman EM. Ubiquitin, the proteasome and protein degradation in neuronal function and dysfunction. Nat Rev Neurosci. 2008; 9:826-38.

[3] Marino G, Madeo F, Kroemer G. Autophagy for tissue homeostasis and neuroprotection. Curr Opin Cell Biol. 2011; 23:198-206.

[4] Ghavami S, Shojaei S, Yeganeh B, Ande SR, Jangamreddy JR, Mehrpour M, et al. Autophagy and apoptosis dysfunction in neurodegenerative disorders. Prog Neurobiol. 2014; 112:24-49.

[5] Dennissen FJ, Kholod N, van Leeuwen FW. The ubiquitin proteasome system in neurodegenerative diseases: culprit, accomplice or victim? Prog Neurobiol. 2012; 96:190-207.

[6] Ying Z, Wang $\mathrm{H}$, Wang $\mathrm{G}$. The ubiquitin proteasome system as a potential target for the treatment of neurodegenerative diseases. Curr Pharm Des. 2013; 19:3305-14.

[7] Diskin T, Tal-Or P, Erlich S, Mizrachy L, Alexandrovich A, Shohami $\mathrm{E}$, et al. Closed head injury induces upregulation of Beclin 1 at the cortical site of injury. J Neurotrauma. 2005; 22:750-62.

[8] Lai Y, Hickey RW, Chen Y, Bayir H, Sullivan ML, Chu CT, et al. Autophagy is increased after traumatic brain injury in mice and is partially inhibited by the antioxidant gamma-glutamylcysteinyl ethyl ester. J Cereb Blood Flow Metab. 2008; 28:540-50.

[9] Liu CL, Chen S, Dietrich D, Hu BR. Changes in autophagy after traumatic brain injury. J Cereb Blood Flow Metab. 2008; 28:674-83.

[10] Kanno H, Ozawa H, Sekiguchi A, Itoi E. The role of autophagy in spinal cord injury. Autophagy. 2009; 5:390-2.

[11] Rodriguez-Muela N, Boya P. Axonal damage, autophagy and neuronal survival. Autophagy. 2012; 8:286-8.

[12] Egami Y, Kiryu-Seo S, Yoshimori T, Kiyama H. Induced expressions of Rab24 GTPase and LC3 in nerve-injured motor neurons. Biochem Biophys Res Commun. 2005; 337:1206-13.
[13] Huang HC, Chen L, Zhang HX, Li SF, Liu P, Zhao TY, et al. Autophagy promotes peripheral nerve regeneration and motor recovery following sciatic nerve crush injury in rats. J Mol Neurosci. 2016; 58:416-23.

[14] Savedia S, Kiernan JA. Increased production of ubiquitin mRNA in motor neurons after axotomy. Neuropathol Appl Neurobiol. 1994; 20:577-86.

[15] Lee HK, Shin YK, Jung J, Seo SY, Baek SY, Park HT. Proteasome inhibition suppresses Schwann cell dedifferentiation in vitro and in vivo. Glia. 2009; 57:1825-34

[16] Hu J, Fink D, Mata M. Microarray analysis suggests the involvement of proteasomes, lysosomes, and matrix metalloproteinases in the response of motor neurons to root avulsion. Eur J Neurosci. 2002; 16:1409-16.

[17] Yang Y, Xie Y, Chai H, Fan M, Liu S, Liu H, et al. Microarray analysis of gene expression patterns in adult spinal motoneurons after different types of axonal injuries. Brain Res. 2006; 1075:1-12.

[18] Agthong S, Kaewsema A, Tanomsridejchai N, Chentanez V. Activation of MAPK ERK in peripheral nerve after injury. BMC Neurosci. 2006; 7:45.

[19] Bramhall S, Noack N, Wu M, Loewenberg JR. A simple colorimetric method for determination of protein. Anal Biochem. 1969; 31:146-8.

[20] Berliocchi L, Russo R, Maiaru M, Levato A, Bagetta G, Corasaniti MT. Autophagy impairment in a mouse model of neuropathic pain. Mol Pain. 2011; 7:83

[21] Zhang E, Yi MH, Ko Y, Kim HW, Seo JH, Lee YH, et al. Expression of LC3 and Beclin 1 in the spinal dorsal horn following spinal nerve ligation-induced neuropathic pain. Brain Res. 2013; 1519:31-9.

[22] Maday S. Mechanisms of neuronal homeostasis: Autophagy in the axon. Brain Res. 2016; 1649:143-50.

[23] Jang SY, Shin YK, Park SY, Park JY, Lee HJ, Yoo YH, et al. Autophagic myelin destruction by Schwann cells during Wallerian degeneration and segmental demyelination. Glia. 2016; 64:730-42. 